\title{
Optical and magnetic properties of Ni nanoparticles in rutile formed by $\mathrm{Ni}$ ion implantation
}

\author{
S. Zhu and L. M. Wang ${ }^{\text {a) }}$ \\ Department of Nuclear Engineering and Radiological Sciences, Department of Materials Science and \\ Engineering, University of Michigan, Ann Arbor, Michigan 48109-2104 \\ X. T. Zu and X. Xiang \\ Department of Applied Physics, University of Electronic Science and Technology of China, Chengdu \\ 610054, People's Republic of China
}

(Received 3 August 2005; accepted 1 December 2005; published online 25 January 2006)

\begin{abstract}
Crystalline Ni nanoparticles in the near surface of $\mathrm{TiO}_{2}$ (rutile) have been synthesized by Ni ion beam implantation at room temperature to a fluence of $1 \times 10^{17} / \mathrm{cm}^{2}$. Transmission electron microscopy, optical absorption spectroscopy, and a superconducting quantum interference device magnetometer have been utilized to characterize the nanostructure, optical and magnetic properties of $\mathrm{Ni}$ particles in $\mathrm{TiO}_{2}$. Crystalline Ni nanoparticles with dimensions ranging 3-20 nm formed in the near surface of rutile, which caused a broad absorption band from $700 \mathrm{~nm}$ in the optical absorption spectrum. Magnetic measurement indicated that the coercive force of Ni nanoparticles was about $210 \mathrm{Oe}$ at $10 \mathrm{~K}$. The superparamagnetism of the nanoparticles was observed above blocking temperature $T=85$ K. C 2006 American Institute of Physics. [DOI: 10.1063/1.2168037]
\end{abstract}

There has been a great deal of recent interest in the incorporation of nanoparticles into dielectric and semiconductor materials to form nanocomposites. Metallic nanoparticles in dielectric matrix have applications for nonlinear optics and ferromagnetic nanoparticles have potential application in magnetic storage devices. ${ }^{1,2}$ Ion implantation is one of the most promising methods for developing such nanocomposites because the implanted species frequently precipitate as clusters or nanoparticles in controlled concentrations at precalculated depths of the host matrix. ${ }^{2}$ A variety of ions have been implanted into diverse matrices, such as $\mathrm{SiO}_{2}, \mathrm{Al}_{2} \mathrm{O}_{3}$, yttria-stabilized zirconia, etc., to form nanocomposites. ${ }^{3-6}$ Nano-crystalline particles incorporated in titanium dioxide $\left(\mathrm{TiO}_{2}\right)$ by ion implantation have attracted considerable attention during the past decade. Nano-sized Au particles synthesized in $\mathrm{TiO}_{2}$ by ion implantation showed unusual nonlinear optical properties enhanced by surface plasmon resonance. ${ }^{7-9}$ A number of metallic ions ( $\mathrm{Cu}, \mathrm{Ag}$, and $\mathrm{Au}$ ) have been doped in $\mathrm{TiO}_{2}$ to form nanoparticles modifying the photocatalytic properties of $\mathrm{TiO}_{2}$ single crystals or thin films. ${ }^{10}$ Room temperature ferromagnetic properties of Co-doped $\mathrm{TiO}_{2}$ have been reported. ${ }^{11}$ In this study, a high dose of $\mathrm{Ni}^{+}$ions was implanted into $\mathrm{TiO}_{2}$ single crystals to form magnetic nanocomposites consisting $\mathrm{Ni}$ nanoparticles in $\mathrm{TiO}_{2}$ matrix for the potential application as magnetic recording device. The microstructures, optical and magnetic properties of the nanocomposites were characterized by transmission electron microscopy (TEM), optical absorption spectroscopy and magnetic spectrometer.

Well-polished (001) oriented $\mathrm{TiO}_{2}$ (rutile) single crystals were implanted with $64 \mathrm{keV} \mathrm{Ni}{ }^{+}$ions at the room temperature to a fluence of $1 \times 10^{17} \mathrm{ions} / \mathrm{cm}^{2}$ using a metal vapor vacuum arc (MEVVA) 80-10 implanter. The maximum temperature during the implantation was monitored to be less than $140{ }^{\circ} \mathrm{C}$. The projected range of $\mathrm{Ni}^{+}$ions in rutile is

a) Author to whom correspondence should be addressed; electronic mail:
lmwang@umich.edu $\sim 70 \mathrm{~nm}$ according to a calculation using SRIM 2000. ${ }^{12} \mathrm{~A}$ JEM 2010FEG/STEM field emission scanning transmission electron microscope operated at $200 \mathrm{keV}$ was used to characterize the depth dependent microstructure of $\mathrm{TiO}_{2}$ after ion implantation in cross section. ${ }^{13}$ The lens conditions of the electron microscope were adjusted for a probe size of $0.2 \mathrm{~nm}$ and with a collection angle of $50 \mathrm{mrad}$ for the high angle annular dark filed (HAADF) Z-contrast STEM imaging. Optical absorption measurements in the ultraviolet visible (at wavelengths of 190-1010 nm) were performed using a SHIMADZU UV-2550 spectrophotometer. The magnetic properties of the samples were characterized with a magnetic property measurement system equipped with a superconducting quantum interference device magnetometer by Quantum Designs at 10 and $300 \mathrm{~K}$, respectively. Zero-field-cooling (ZFC) and field-cooling (FC) magnetizations as a function of temperature were measured under an applied magnetic field of 100 Oe.

Nanoparticles of $\mathrm{Ni}$ precipitated spontaneously during ion implantation from the surface of $\mathrm{TiO}_{2}$ matrix to a depth of $50 \mathrm{~nm}$, as revealed by the cross-sectional HAADF Z-contrast image shown in Fig. 1(a). In a HAADF STEM image, the intensity of a local area is approaching a $Z^{2}$ dependence on atomic number $\mathrm{Z}$, suggesting a HAADF image provides chemical information by its contrast (so called $\mathrm{Z}$-contrast image). Because $\mathrm{Ni}$ has a higher $\mathrm{Z}$ than both $\mathrm{Ti}$ and $\mathrm{O}$, the nanoparticles of $\mathrm{Ni}$ show a brighter contrast in Fig. 1(a). The dimensions of nanoparticles ranged from 3 to $10 \mathrm{~nm}$. Some elongated precipitates up to $20 \mathrm{~nm}$ in length were observed in the near surface of $\mathrm{TiO}_{2}$. These elongated $\mathrm{Ni}$ precipitates are observed with the round shape in a planview TEM image. The implantation region of $\mathrm{TiO}_{2}$ matrix has been damaged and amorphized to $60 \mathrm{~nm}$ in the depth below the surface. Figure 1(b) is a high resolution TEM (HRTEM) image of Ni nanoparticles embedded in the $\mathrm{TiO}_{2}$ matrix, revealing a well-developed crystalline structure of $\mathrm{Ni}$ nanoparticles after ion implantation without a thermal treatment. 

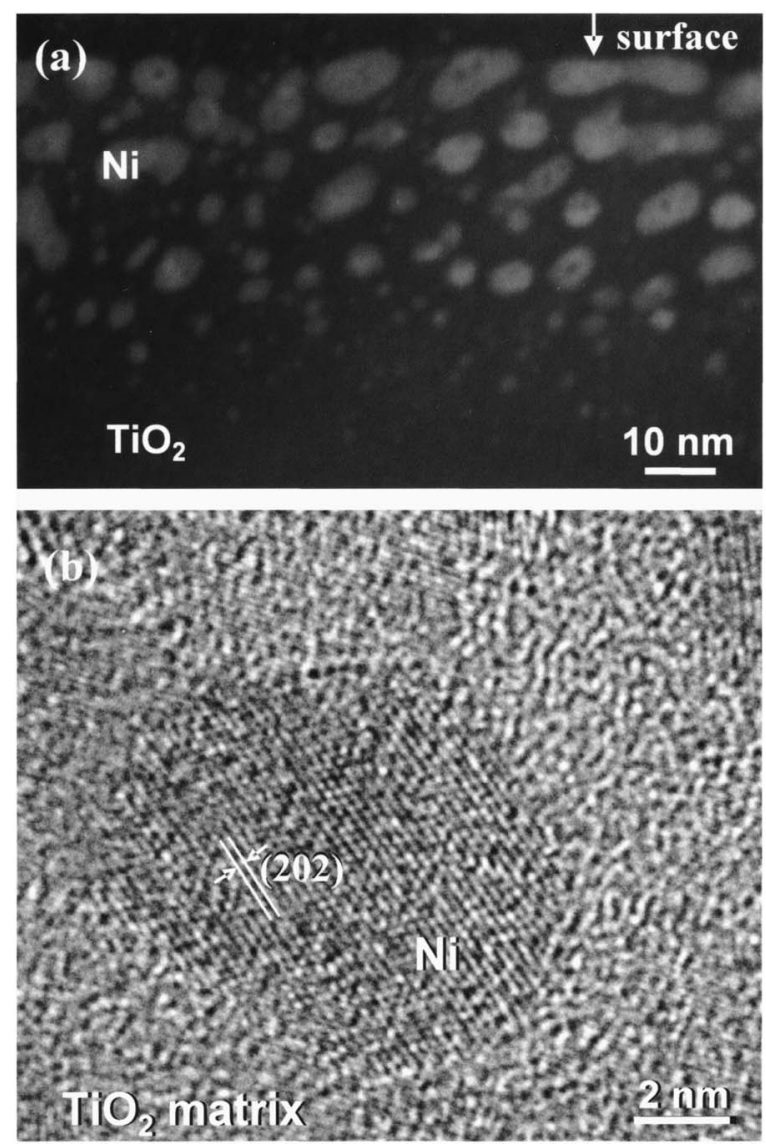

FIG. 1. A cross-sectional Z-contrast STEM image of Ni nanoparticles in the near surface of a $\mathrm{TiO}_{2}$ single crystal (a); and a HRTEM micrograph showing a crystalline $\mathrm{Ni}$ nanoparticle in the amorphous $\mathrm{TiO}_{2}$ matrix (b).

The optical absorption spectrum of $\mathrm{TiO}_{2}$ single crystals after Ni ion implantation is shown in Fig. 2. The absorption spectrum of the un-implanted sample is also shown for comparison. As shown in the figure, the absorption in the UV region is the same between the as-grown and the $\mathrm{Ni}^{+}$-implanted $\mathrm{TiO}_{2}$, with a well-known absorption edge at $410 \mathrm{~nm}$ (the fundamental band gap $3.0 \mathrm{eV}$ of rutile $\mathrm{TiO}_{2}$ ). The absorption intensity of the implanted sample was enhanced distinctively in the visible-near IR region due to im-

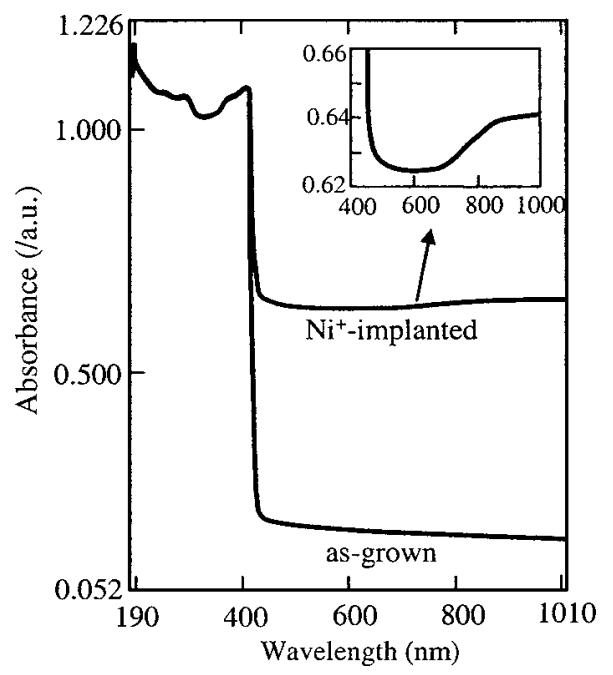

FIG. 2. Optical absorption spectra of Ni-implanted $\mathrm{TiO}_{2}$ and as-grown $\mathrm{TiO}_{2}$ single crystals.

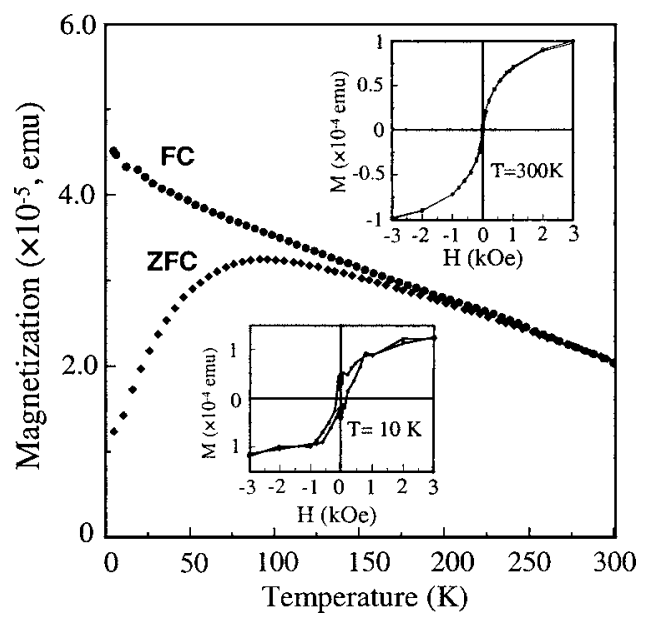

FIG. 3. Temperature dependence of the ZFC and FC magnetization curves for the $\mathrm{Ni}$ implanted $\mathrm{TiO}_{2}$ sample with magnetic hysteresis loops at $10 \mathrm{~K}$ (bottom inset) and $300 \mathrm{~K}$ (top inset).

plantation damage. In addition, a very broad absorption band appears from $700 \mathrm{~nm}$ to the instrument detection limit (1010 $\mathrm{nm})$. This change may be attributed to the Ni nanoparticles' formation in rutile. In previous studies, ${ }^{10} \mathrm{Cu}$-implanted rutile (100) single crystals showed a small peak or shoulder near $2.05 \mathrm{eV}(605 \mathrm{~nm})$, and Ag-implanted samples showed an absorption peak near $2.5 \mathrm{eV}(496 \mathrm{~nm})$ due to the surface plasmon resonance (SPR) absorption. In those conditions, the size of nanoparticles for both $\mathrm{Cu}$ and $\mathrm{Ag}$ was considered to be very small, approximately $1-2 \mathrm{~nm}$. Thus the broadband shown in our Ni-implanted sample must be due to the surface plasmon resonance (SPR) absorption and the broad size distribution of $\mathrm{Ni}$ nanoparticles in rutile $\mathrm{TiO}_{2}$.

Optical absorption properties of $\mathrm{TiO}_{2}$ with metal nanoparticles in a surface layer within $30 \mathrm{~nm}$ in depth were usually estimated by Mie's scattering theory. As is shown by Oshchephov and Sinyuke, ${ }^{14}$ where the particles' size is smaller than Rayleigh limit of Mie's theory, the maximum position and the shape of extinction spectrum are entirely independent of particle size but rather depend only on the optical constants of the particle material and those of the surrounding medium. However, for larger particle sizes, both the peak position and the width of the extinction spectrum are strongly dependent on particle size. In the $\mathrm{Ni}^{+}$-implanted rutile sample, the size of $\mathrm{Ni}$ nanoparticles ranges from 3 to $20 \mathrm{~nm}$, which results in a broad absorption band and an uncertain peak position. In addition, the irregular shape of $\mathrm{Ni}$ nanoparticles may have also contributed to this broad absorption band.

Figure 3 shows the zero-field-cooling (ZFC) and fieldcooling (FC) magnetization as a function of temperature of $\mathrm{Ni}$ nanoparticles in $\mathrm{TiO}_{2}$ matrix. ZFC and $\mathrm{FC}$ curves were measured by cooling the sample initially in a zero field to 10 $\mathrm{K}$, and magnetization was recorded in an applied magnetic field where $H=100 \mathrm{Oe}$ as the temperature increased. When the temperature reached $300 \mathrm{~K}$, the sample was gradually cooled, and the magnetization was recorded. Figure 3 clearly shows the nonzero difference between the FC and ZFC data, indicating the hysteresis while eliminating any para- and diamagnetic contributions. In these ZFC/FC curves, the temperature at the position with maximum magnetization is known as the blocking temperature, $T_{B}$, which is $\sim 85 \mathrm{~K}$. Above the blocking temperature, the magnetization is un- 


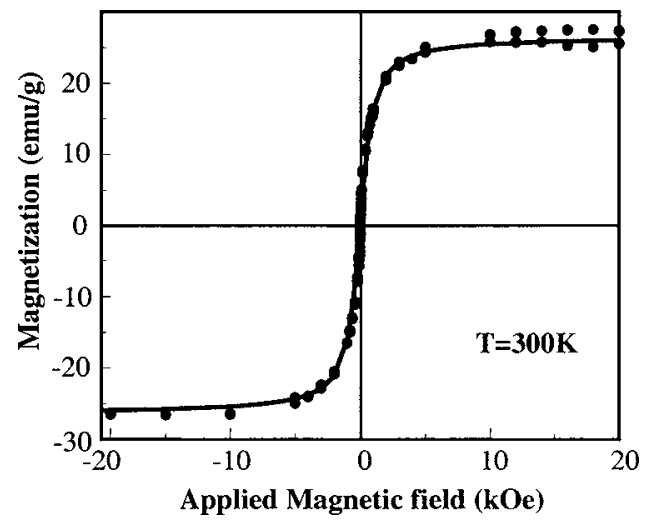

FIG. 4. Measured (solid circle) and the Langevin function fitted (solid line) magnetization $(M)$ vs magnetic field $(H)$ at the room temperature.

stable and the sample loses all its hysteric responses. The two insets in Fig. 3 show magnetization plots as a function of magnetic field $(M-H)$ at 10 and $300 \mathrm{~K}$, respectively. The applied magnetization field $H$ is parallel to (100) of the $\mathrm{TiO}_{2}$ single crystal. The magnetic hysteresis loop at $10 \mathrm{~K}$ after correcting paramagnetic contribution from $\mathrm{TiO}_{2}$ matrix shows a ferromagnetic behavior with coercivity, $H_{c}$, equal to $\sim 210$ Oe. This value is larger than that of bulk Ni (176 Oe) and of Ni nanoparticles in $\mathrm{MgO}$ (195 Oe). ${ }^{15,16}$ This large coercivity can be explained by the nano-size effect and the single domain structure (the critical size of the single domain for spherical Ni particles was $\sim 42 \mathrm{~nm}$ ). ${ }^{17,18}$ The magnetization curves at $10 \mathrm{~K}$ were completely saturated at applied fields $H=4000$ Oe. The remnant magnetization of the saturation magnetization $\left(M_{r} / M_{s}\right)$ is about $40 \%$ at this temperature. The coercive force and remnant magnetization of $\mathrm{Ni}$ nanoparticles were not observed as the temperature increased to $300 \mathrm{~K}$, confirming the superparamagnetic behavior of the nanoparticles above the blocking temperature. Since some nanoparticles are not truly spherical, the shape anisotropy contributes to the superparamagnetism.

Langevin function can be used to calculate the true magnetic moment of each particle for superparamagnetic particles as ${ }^{19}$

$$
M(H / T)=M_{0}\left[\operatorname{coth}\left(M_{0} m H / k_{B} T\right)-\left(k_{B} T / M_{0} m H\right)\right],
$$

where $M_{0}$ is the saturation magnetization $(\mathrm{emu} / \mathrm{g}), m$ is the mass of individual particle (gram), and $k_{B}$ is Boltzmann constant. The saturation magnetization in our sample was about $25 \mathrm{emu} / \mathrm{g}$ from the hysteresis loop at $300 \mathrm{~K}$. Figure 4 shows magnetization versus applied magnetic field of Ni nanoparticles at $300 \mathrm{~K}$ (solid circles) and the best fit for the Langevin function (solid line). From the data fitting, we calculate the average grain size of $9.4 \mathrm{~nm}$ for the Ni nanoparticles contributing to magnetic moment, which is in the range of particle size measured by TEM observation. The mean-magnetic moment per particle of the sample was calculated to be $11064 \mu_{B}$.
In conclusion, $\mathrm{Ni}$ nanocrystals with $3-20 \mathrm{~nm}$ in dimensions embedded in $\mathrm{TiO}_{2}$ (rutile) matrix have been synthesized by ion beam implantation. A new broad absorption band from $700 \mathrm{~nm}$ appeared in the optical absorption spectrum of the $\mathrm{Ni}^{+}$-implanted $\mathrm{TiO}_{2}$ which may have resulted from the SPR absorption of Ni nanoparticles in the surface of $\mathrm{TiO}_{2}$. The broadening of the peak is owing to the wide size distribution of nanoparticles. The coercivity of Ni nanoparticles was about 210 Oe at $10 \mathrm{~K}$. Superparamagnetic property of Ni nanoparticles was characterized, showing a blocking temperature of $85 \mathrm{~K}$. Fitting the Langevin function at 300 $\mathrm{K}$, an effective average particle size of $9.4 \mathrm{~nm}$ contributing to the magnetic moment was obtained.

The TEM analysis was conducted in the Electron Microbeam Analysis Laboratory at the University of Michigan, and we acknowledge the support of NSF award No. DMR9371177 for the electron microscope. This work is supported by U.S. Department of Energy under Grant No. DE-FG0202ER46005, and the NSAF Joint Foundation of China (10376006).

${ }^{1}$ L. L. Beecroft and C. K. Ober, Chem. Mater. 9, 1302 (1997).

${ }^{2}$ A. Meldrum, L. A. Boatner, and C. W. White, Nucl. Instrum. Methods Phys. Res. B 178, 7 (2001).

${ }^{3}$ K. Fukumi, A. Chayahara, K. Kadono, T. Sakaguchi, Y. Horino, M. Miya, K. Fujii, J. Hayakawa, and M. Satou, J. Appl. Phys. 75, 3075 (1994).

${ }^{4}$ S. Zhu, K. Sun, Q. Y. Zhang, X. T. Zu, L. M. Wang, and R. C. Ewing, J. Appl. Phys. 94, 5648 (2003).

${ }^{5}$ X. Xiang, X. T. Zu, S. Zhu, and L. M. Wang, Appl. Phys. Lett. 84, 52 (2004).

${ }^{6}$ X. Xiang, X. T. Zu, J. W. Bao, S. Zhu, and L. M. Wang, J. Appl. Phys. 98, 073524 (2005).

${ }^{7}$ S. Nakao, T. Nonami, P. Jin, Y. Miyagawa, and S. Miyagawa, Surf. Coat. Technol. 128, 446 (2000).

${ }^{8}$ K. Sun, S. Zhu, R. Fromknecht, G. Linker, and L. M. Wang, Mater. Lett. 58, 547 (2004).

${ }^{9}$ S. Zhu, K. Sun, L. M. Wang, R. C. Ewing, and R. Fromknecht, Nucl. Instrum. Methods Phys. Res. B 242, 152 (2006).

${ }^{10}$ H. Tsuji, T. Sagimori, K. Kurita, Y. Gotoh, and J. Ishikawa, Surf. Coat. Technol. 158,159, 208 (2002).

${ }^{11}$ V. Shuttanandan, S. Thevuthasan, S. M. Heald, T. Droubay, M. H. Engelhard, T. C. Kaspar, D. E. McCready, L. Saraf, S. A. Chambers, B. S. Mun, N. Hamdan, P. Nachimuthu, B. Taylor, R. P. Sears, and B. Sinkovic, Appl. Phys. Lett. 84, 4466 (2004).

${ }^{12}$ J. F. Ziegler, The Stopping and Range of Ions in Matter (IBM-Research, Yorktown, NY, 2000).

${ }^{13}$ L. M. Wang, Nucl. Instrum. Methods Phys. Res. B 141, 312 (1998).

${ }^{14}$ S. L. Oshchephov and A. F. Sinyuk, J. Colloid Interface Sci. 208, 137 (1998).

${ }^{15}$ P. Saravanan, T. A. Jose, P. J. Thomas, and G. U. Kulkarni, Bull. Mater. Sci. 24, 515 (2001).

${ }^{16}$ S. Zhu, X. Xiang, X. T. Zu, and L. M. Wang, Nucl. Instrum. Methods Phys. Res. B 242, 114 (2005).

${ }^{17}$ Y. W. Du, M. X. Xu, J. Wu, Y. B. Shi, and H. X. Lu, J. Appl. Phys. 70, 5903 (1991).

${ }^{18}$ Z. J. Zhao, H. Q. Wang, B. Wang, J. G. Hou, G. L. Liu, and X. F. Jin, Phys. Rev. B 65, 235413 (2002).

${ }^{19}$ J. P. Bucher, D. C. Douglass, and L. A. Bloomfield, Phys. Rev. Lett. 66, 3052 (1991). 